\title{
The relationship between vitamin D level and hepatosteatosis in obese children
}

\author{
(D) Fatma Dursun, ${ }^{1}$ (D) Nelgin Gerenli, ${ }^{2}$ (D) Seyma Meliha Su Dur, ${ }^{3}$ (D) Heves Kirmizibekmez ${ }^{1}$ \\ ${ }^{1}$ Department of Pediatric Endocrinology, Umraniye Training and Research Hospital, Istanbul, Turkey \\ 2Department of Pediatric Gastroenterology, Umraniye Training and Research Hospital, Istanbul, Turkey \\ ${ }^{3}$ Department of Radiology, Umraniye Training and Research Hospital, Istanbul, Turkey
}

\begin{abstract}
OBJECTIVE: The increasing incidence of obesity in children is a significant risk factor for nonalcoholic fatty liver disease and obesity-associated morbidity. In the present study, we aimed to explore the correlation between Vitamin D level and hepatosteatosis in obese children.

METHODS: A total of 110 children aged 10-16 years who presented to pediatric endocrinology outpatient clinic for obesity were enrolled. The study was completed in a single season between September and November. Hepatosteatosis was diagnosed by ultrasonography. The patients were grouped into two groups: Group 1 comprised patients with hepatosteatosis and Group 2 consisted of patients without hepatosteatosis. 25 hydroxy $(25-\mathrm{OH})$ Vitamin D levels were compared between patients with and without hepatosteatosis.

RESULTS: No statistically significant difference was observed between 25-OH Vitamin D levels of patients with and without hepatosteatosis. When the effects of age and sex were kept constant, there was no significant correlation between Vitamin D level and aspartate aminotransferase, alanine aminotransferase, and body mass index values.

CONCLUSION: Unlike the results of the previous studies, we were unable to detect any significant difference between Vitamin D levels of obese patients with and without hepatosteatosis. We think that obesity, rather than Vitamin D status, that is, in fact, independently associated with nonalcoholic fatty liver disease. Larger studies are needed to investigate the impact of Vitamin D in children with obesity with hepatosteatosis.
\end{abstract}

Keywords: Hepatosteatosis; obese children; Vitamin D.

Cite this article as: Dursun F, Gerenli N, Su Dur SM, Kirmizibekmez H. The relation between vitamin D level and hepatosteatosis in obese children. North Clin Istanb 2019;6(1):28-32.

$\mathrm{O}$ besity is a significant health problem affecting an increasing number of children worldwide and has been reported an epidemic by the World Health Organization $[1,2]$. As with obesity, Vitamin D deficiency is reaching epidemic proportions worldwide, in both pediatric and adult populations [3]. Reports to date have linked Vitamin D deficiency to hypertension, diabetes mellitus, and insulin resistance (IR), nonalcoholic fatty liver disease (NAFLD), and metabolic syndrome [4-6].
NAFLD has become the most common form of the chronic liver disease with a prevalence as high as $30 \%$, $[7,8]$ thus exceeding that of alcoholic liver disease and viral hepatitis. NAFLD means a continuum of hepatic injuries, which progress from simple fatty liver to steatohepatitis (NASH), cirrhosis, or even hepatocellular carcinoma. The metabolic syndrome is worldwide considered as the key factor in the pathogenesis of NAFLD [9]. However, the evolution of liver inflammation in NAFLD

Received: October 25, 2017 Accepted: May 16, 2018 Online: August 08, 2018

Correspondence: Dr. Fatma DURSUN. Umraniye Egitim ve Arastirma Hastanesi, Pediatrik Endokrinoloji Klinigi, Istanbul, Turkey. 
and the progression from simple fatty liver to steatohepatitis and hepatic fibrosis are more complex. Insulin resistance, a key risk factor in the pathogenesis of NAFLD, is linked to the development of oxidative stress and lipotoxicity [10].

Given the strong association of NAFLD with obesity and the metabolic syndrome, recent years there has been a scientific interest into the potential role of Vitamin $\mathrm{D}$ in NAFLD. Vitamin D deficiency is associated with NAFLD and has been correlated with disease severity $[10,11]$. However, a recent Korean study showed contradictory results. In this study, there was no significant difference in Vitamin D levels between groups [12]. An another study, in the National Health and Nutrition Examination Survey from 2001 to 2004, suspected NAFLD, which was defined by elevated serum alanine aminotransferase ([ALT]; >30 U/L), was not associated with Vitamin D status in adolescents after adjustment for obesity [13]. Li et al. [14] showed that serum 25 hydroxy $(25[\mathrm{OH}])$ D concentrations or Vitamin D status were not significantly associated with the presence of NAFLD. Thus, the role of Vitamin D in the pathogenesis of NAFLD remains unclear. In the present study, we explored whether Vitamin D levels were different in patients with and without NAFLD.

\section{MATERIALS AND METHODS}

This prospective study involved 110 patients aged 1016 years who presented to the pediatric endocrinology outpatient clinic for obesity in the same season between September and November.

The patients were grouped into two groups: Group 1 comprised patients with hepatosteatosis and Group 2 consisted of patients without hepatosteatosis. Exclusion criteria were hypothyroidism, HBV or HCV infection, a-1-antitrypsin deficiency, Wilson's disease, use of known steatogenic drugs, and another chronic disease. Patients who had received vitamin supplementation in the past 6 months that might have potentially changed Vitamin D level were also excluded from the study. Ethical approval was obtained from the hospital Ethics Committee, and written informed consent was obtained from each of the parents or legal guardians of the patients. Children with a body mass index $(\mathrm{BMI})>95^{\text {th }}$ percentile were diagnosed as having obesity [15]. BMI was calculated as weight (kilograms)/height (meter) 2, evaluated against the BMI reference percentiles for Turkish children [16]. All blood samples were collected in the morning after an overnight fast. Serum levels of vitamin D may have seasonal variability; [17] therefore, all blood samples were taken in the same season.

We used the homeostasis model of assessment (HOMA) to calculate IR in children with obesity, which was calculated as follows: HOMA-IR; fasting glucose $(\mathrm{mg} / \mathrm{dL}) \times$ fasting insulin $(\mathrm{mU} / \mathrm{mL}) / 405$ [18].

Laboratory tests included the following: ALT, aspartate aminotransferase (AST), calcium, phosphate, alkaline phosphatase, 25-OH Vitamin $\mathrm{D}$, parathyroid hormone (PTH), triglyceride, total cholesterol, low-density lipoprotein, high-density lipoprotein, blood glucose, and insulin. Glucose, ALT, and AST levels were studied using Abbott kits and an Abbott i8000 model device at our hospital's central laboratory. Glucose level was studied using the Hexokinase/G-6-PDH Method; ALT and AST were measured with the enzymatic method. Insulin, PTH, and 25OHD3 levels were measured with an Abbott 116000 model device using the "chemiluminescent microparticle immunoassay" method and Abbott kits.

\section{Liver ultrasonography}

Ultrasound imaging can be used as a sensitive method for diagnosing steatosis of liver [19]. Assessment of hepatosteatosis was carried out by an expert from the radiology department using Toshiba iStyle Aplio MX model with $3.5 \mathrm{MHz}$ convex probes. All ultrasound examinations were performed by the single experienced pediatric radiologist. Absent steatosis (Grade 0) was defined as normal liver echo-texture; mild steatosis (Grade 1) as slight and diffuse increase in fine parenchymal echoes with normal visualization of diaphragm and portal vein borders; moderate steatosis (Grade 2) as moderate and diffuse increase in fine echoes with slightly impaired visualization of diaphragm and portal vein borders; and severe steatosis (Grade 3) as fine echoes with poor or no visualization of diaphragm, portal vein borders, and posterior portion of the right lobe $[20,11]$.

\section{Statistical analysis}

Study data were analyzed using IBM SPSS Statistics 22 (IBM SPSS, Turkey) software package. Shapiro-Wilk test was used to test the normality of study data. Descriptive statistics included mean and standard deviation. Quantitative variables with normal distribution were compared using Student t-test between the two groups and Mann-Whitney U-test was used to compare quantitative variables without a normal distribution. Conti- 
nuity (Yates) correction was used for the comparison of qualitative data. Pearson correlation analysis has been used to analyze the correlation between the parameters showing normal distribution, whereas Spearman's rho correlation analysis has been used to analyze the parameters not showing normal distribution. For all statistical procedures, the significance level was set at $\mathrm{p}<0.05$.

\section{RESULTS}

In this study 110 children with obesity were enrolled, of whom $55.5 \%(n=61)$ were girls; mean age of the study population was $12.19 \pm 2.5$ years $(10-16$ years). 32 (29. $1 \%)$ patients were diagnosed with hepatosteatosis. 15 of 32 patients had Grade 1 hepatosteatosis and 17 had
Grade 2 hepatosteatosis. The clinical and laboratory characteristics of the study population are summarized in Table 1 and 2.

Patients with NAFLD (Group 1) had significantly greater BMI than those without (Group 2) $(\mathrm{p}<0.05)$. Patients with NAFLD had a significantly greater HOMA-IR than those without $(\mathrm{p}<0.05)$. There was no statistically significant difference in the incidence of IR when NAFLD patients were compared with those without NAFLD ( $>00.05)$. Of the 32 patients with NAFLD, $38.7 \%$ (12 patients) had IR, while 78 patients without NAFLD, 28.2\% (22 patients) IR were found in them. Patients with NAFLD had a significantly greater AST level than those without $(\mathrm{p}<0.01)$. Patients with NAFLD had a significantly greater ALT level than those

TABLE 1. Anthropometric and clinical data of children with hepatosteatosis (Group 1) and without hepatosteatosis (Group 2)

\begin{tabular}{lcccc} 
& Group 1 & Group 2 & All cases & $\mathrm{p}$ \\
\hline Age (years) & $12.71 \pm 2.38$ & $11.97 \pm 2.64$ & $12.19 \pm 2.58$ & 20.177 \\
Female, $\mathrm{n}(\%)$ & $15(46.9)$ & $46(59)$ & $61(55.4)$ & 0.343 \\
Male, $\mathrm{n}(\%)$ & $17(53.1)$ & $32(41)$ & $49(44.5)$ & \\
BMI $\left(\mathrm{kg} / \mathrm{m}^{2}\right)$ & $33.61 \pm 14.82$ & $29.86 \pm 4.58$ & $30.92 \pm 8.88$ & ${ }^{20.046^{*}}$ \\
\hline
\end{tabular}

${ }^{2}$ Student $\mathrm{t}$ test; ${ }^{*} \mathrm{p}<0.05$; BMI: Body mass index.

TABLE 2. Laboratory data of children with hepatosteatosis (Group 1) and without hepatosteatosis (Group 2)

\begin{tabular}{|c|c|c|c|c|}
\hline AST (IU/L) & $38.52 \pm 46.97(28)$ & $22.11 \pm 10.9(20)$ & $26.86 \pm 27.65(21)$ & $10.001 * *$ \\
\hline $25-\mathrm{OH} D(\mathrm{ng} / \mathrm{mL})$ & $17.4 \pm 5.74$ & $16.85 \pm 5.98$ & $17.01 \pm 5.89$ & 20.659 \\
\hline Calcium (mg/dL) & $9.98 \pm 0.29$ & $9.77 \pm 0.35$ & $9.81 \pm 0.35$ & 20.063 \\
\hline Phosphate (mg/dL) & $4.87 \pm 0.49$ & $4.64 \pm 0.68$ & $4.69 \pm 0.65$ & 20.363 \\
\hline $\mathrm{PTH}(\mathrm{pg} / \mathrm{mL})$ & $45.7 \pm 12.5$ & $42.4 \pm 21.3$ & $43.8 \pm 13.5$ & 0.88 \\
\hline HOMA-IR & $4.97 \pm 2.77$ & $3.91 \pm 2.04$ & $4.22 \pm 2.31$ & $20.029 *$ \\
\hline Triglyceride $(\mathrm{mg} / \mathrm{dL})$ & $133.06 \pm 66.49$ & $125.83 \pm 54.87$ & $128.01 \pm 58.37$ & 20.567 \\
\hline $\mathrm{HDL}(\mathrm{mg} / \mathrm{dL})$ & $41.88 \pm 12.82$ & $41.2 \pm 7.41$ & $41.0 \pm 9.28$ & 20.731 \\
\hline $\mathrm{LDL}(\mathrm{mg} / \mathrm{dL})$ & $109.55 \pm 31.49$ & $105.43 \pm 25.73$ & $106.71 \pm 27.55$ & 20.493 \\
\hline
\end{tabular}

${ }^{1}$ Mann Whitney $\mathrm{U}$ test; ${ }^{2}$ Student $t$ test; ${ }^{*} \mathrm{p}<0.05 ;{ }^{* *} \mathrm{p}<0.01$; AST: Aspartate aminotransferase; ALT: Alanine aminotransferase; 25 OH vit D: 25 hydroxy vitamin D3; ALP: Alkaline phosphatase; HOMA-IR: Homeostasis model of assessment insulin resistance; HDL: High-density lipoprotein; LDL: Low-density lipoprotein; PTH: Parathyroid hormone. 
without $(p<0.01)$. There is a $24.9 \%$ positive statistically significant relationship between the HOMA-IR and hepatosteatosis level $(\mathrm{p}<0.01$, rho=0.249).

On the other hand, no significant difference was found between 25-OH Vitamin D levels of Group 1 and Group 2 ( $p>0.05)$ (Table 2). When the effects of age and sex were kept constant, there was no significant correlation between Vitamin D level and AST, ALT, and BMI values $(p>0.05)$.

\section{DISCUSSION}

According to epidemiological data, NAFLD may affect $3-10 \%$ of pediatric patients, with a male-to-female ratio of $2: 1$ [21]. In our study, $53.1 \%$ of the patients with NAFLD were male. Both NAFLD and Vitamin D deficiency is common in obese subjects. While obesity is a risk factor for both conditions, NAFLD and Vitamin D association is not clear.

Enhanced ALT levels are common among pediatric patients with NAFLD. Aminotransferase levels may range from normal to 4-6 times the upper limit of normal. Mild aminotransferase elevation is usually observed in NAFLD patients [22]. In our study, ALT and AST levels were significantly greater in the group with NAFLD.

NAFLD was initially considered a two-hit process. At present, however, the attitude that NAFLD pathogenesis is multifactorial with many factors affecting disease development and progression is widely accepted. The "multiple-hit" hypothesis is currently the established pathogenetic model. The onset of disease, NAFLD is characterized by fat accumulation in the liver (steatosis) and IR, influenced by genetic susceptibility, epigenetic mechanism, a sedentary lifestyle, and hypercaloric diets [22]. Insulin resistance, a key risk factor in the pathogenesis of NAFLD, is linked to the development of oxidative stress and lipotoxicity [10]. Hepatic triglyceride accumulation proceeds from increased delivery of free fatty acids to the liver increased lipogenesis, and impaired fatty acid metabolism in hepatocytes. Hepatic fat accumulation has been shown to aggravate IR by interfering with phosphorylation of insulin receptor substrates. Free fatty acid accumulation and IR may predispose the fatty liver to secondary hits, including oxidative stress, mitochondrial dysfunction, and pro-inflammatory cytokines imbalance, which lead to necroinflammation [22]. Our study also showed that IR was greater in patients with NAFLD.
While the role of Vitamin D in the regulation of calcium and bone homeostasis is well demonstrated, there is evidence for Vitamin D also having immunomodulatory, anti-fibrotic, and anti-inflammatory properties [23]. In adults and children affected by NAFLD, Vitamin D levels have been reported to be inversely associated with liver steatosis, necroinflammation, and fibrosis. In rats exposed to western-style diets, Vitamin D deficiency exacerbates NAFLD through the activation of the toll-like receptor, possibly by means of endotoxin exposure, causing IR, and upregulation of hepatic inflammatory genes [11]. However, in a recent Korean study contradictory results were obtained. In this study, the authors analyzed data from the Korean National Health and Nutrition Examination database (KNHANES IV) with $>16.000$ individuals and reported that obese subjects $(\mathrm{BMI}>25)$ with $>2$ components of the metabolic syndrome were more likely to have elevated liver enzymes compared to normal weight subjects; however, there was no significant difference in Vitamin D levels between the group [17]. Li et al. [14] showed that serum $25(\mathrm{OH})$ D levels were not significantly associated with the presence of NAFLD. It is well known that adolescents with obesity and its comorbidities are at increased risk for developing NAFLD and that increased BMI is a significant independent predictor of hypovitaminosis D [24]. Low concentrations of serum $25(\mathrm{OH}) \mathrm{D}$ found in obese adolescents may show an inadequate diet or a more sedentary lifestyle with less time spent outdoors, resulting in less sunlight exposure. It also is possible that lower serum $25(\mathrm{OH})$ $\mathrm{D}$ levels in obese individuals' reflect the sequestration of Vitamin D in adipose tissue [25]. Other predictors of hypovitaminosis $\mathrm{D}$ in adolescents may include ethnicity, season, and milk consumption. Considering that adiposity is well documented as contributing to the risk of developing NAFLD, these results suggest that it is obesity, rather than Vitamin D status, that is, in fact, independently associated with NAFLD. Katz et al. [13] showed that suspected NAFLD is not associated with Vitamin D status in adolescents after adjustment for obesity. Our study also failed to demonstrate any significant difference between the vitamin D levels of groups with and without hepatosteatosis.

\section{Conclusion}

We were unable to detect any significant difference between obese patients with and without hepatosteatosis with regard to Vitamin D level. We are of the opinion 
that obesity, rather than vitamin D status, that is, in fact, independently associated with NAFLD. Larger, randomized studies are needed to investigate the impact of Vitamin D in children with obesity with hepatosteatosis.

\section{Conflict of Interest: The authors declare no conflict of interest.}

Financial Disclosure: The authors declared that this study has received no financial support.

Authorship Contributions: Concept - F.D.; Design - F.D., N.G., S.M.S.D.; Supervision - F.D., N.G., H.K.; Materials - F.D., N.G., S.M.S.D.; Data collection \&/or processing - F.D., S.M.S.D., N.G.; Analysis and/or interpretation - F.D., N.G.; Writing - F.D., H.K., S.M.S.D.; Critical review - F.D., H.K., N.G.

\section{REFERENCES}

1. Obesity: Preventing and Managing the Global Epidemic. Report of a WHO consultation. World Health Organ Tech Rep Ser 2000;894:1$12,1-253$.

2. Yıldız I, Erol OB, Toprak S, Cantez MS, Omer B, Kılıç A, et al. Role of Vitamin D in children with hepatosteatosis. J Pediatr Gastroenterol Nutr 2014;59:106-11. [CrossRef]

3. Walker GE, Ricotti R, Roccio M, Moia S, Bellone S, Prodam F, et al. Pediatric obesity and vitamin D deficiency: A proteomic approach identifies multimeric adiponectin as a key link between these conditions. PLoS One 2014;9:e83685. [CrossRef]

4. Ford ES, Ajani UA, McGuire LC, Liu S. Concentrations of serum vitamin D and the metabolic syndrome among U.S. Adults. Diabetes Care 2005;28:1228-30. [CrossRef]

5. Holick MF, Binkley NC, Bischoff-Ferrari HA, Gordon CM, Hanley DA, Heaney RP, et al. Evaluation, treatment, and prevention of vitamin D deficiency: An endocrine society clinical practice guideline.J Clin Endocrinol Metab 2011;96:1911-30. [CrossRef]

6. Zúñiga S, Firrincieli D, Housset C, Chignard N. Vitamin D and the vitamin D receptor in liver pathophysiology. Clin Res Hepatol Gastroenterol 2011;35:295-302. [CrossRef]

7. Lazo M, Clark JM. The epidemiology of nonalcoholic fatty liver disease: A global perspective. Semin Liver Dis 2008;28:339-50. [CrossRef]

8. Browning JD, Szczepaniak LS, Dobbins R, Nuremberg P, Horton JD, Cohen JC, et al. Prevalence of hepatic steatosis in an urban population in the united states: Impact of ethnicity. Hepatology 2004;40:1387-95.

9. Fabbrini E, Sullivan S, Klein S. Obesity and nonalcoholic fatty liver disease: Biochemical, metabolic, and clinical implications. Hepatology 2010;51:679-89. [CrossRef]

10. Eliades M, Spyrou E. Vitamin D: A new player in non-alcoholic fatty liver disease? World J Gastroenterol 2015;21:1718-27. [CrossRef]

11. Nobili V, Giorgio V, Liccardo D, Bedogni G, Morino G, Alisi A, et al. Vitamin D levels and liver histological alterations in children with nonalcoholic fatty liver disease. Eur J Endocrinol 2014;170:547-53. [CrossRef]

12. Hong HC, Lee JS, Choi HY, Yang SJ, Yoo HJ, Seo JA, et al. Liver enzymes and vitamin D levels in metabolically healthy but obese individuals: Korean national health and nutrition examination survey. Metabolism 2013;62:1305-12. [CrossRef]

13. Katz K, Brar PC, Parekh N, Liu YH, Weitzman M. Suspected nonalcoholic fatty liver disease is not associated with vitamin $\mathrm{d}$ status in adolescents after adjustment for obesity. J Obes 2010;2010:496829.

14. Li L, Zhang L, Pan S, Wu X, Yin X. No significant association between Vitamin D and nonalcoholic fatty liver disease in a chinese population. Dig Dis Sci 2013;58:2376-82. [CrossRef]

15. Physical Status: The Use and Interpretation of Anthropometry. Report of a WHO expert committee. World Health Organ Tech Rep Ser 1995;854:1-452.

16. Bundak R, Furman A, Gunoz H, Darendeliler F, Bas F, Neyzi O, et al. Body mass index references for turkish children. Acta Paediatr 2006;95:194-8. [CrossRef]

17. Itoh H, Mori I, Matsumoto Y, Maki S, Ogawa Y. Vitamin D deficiency and seasonal and inter-day variation in circulating 25-hydroxyvitamin $\mathrm{D}$ and parathyroid hormone levels in indoor daytime workers: A longitudinal study. Ind Health 2011;49:475-81. [CrossRef]

18. Matthews DR, Hosker JP, Rudenski AS, Naylor BA, Treacher DF. Homeostasis model assessment: insulin resistance and $B$ cell function from fasting plasma glucose and insulin concentrations in man. Diabetologia 1985;28:412-19. [CrossRef]

19. Saadeh S, Younossi ZM, Remer EM, Gramlich T, Ong JP, Hurley M, et al. The utulity of radiological imaging in nonalcoholic fatty liver disease. Gastroenterology 2002;123:745-50. [CrossRef]

20. Shannon A, Alkhouri N, Carter-Kent C, Monti L, Devito R, Lopez R, et al. Ultrasonographic quantitative estimation of hepatic steatosis in children with NAFLD. J Pediatr Gastroenterol Nutr 2011;53:190-5.

21. Alisi A, Manco M, Vania A, Nobili V. Pediatric nonalcoholic fatty liver disease in 2009. J Pediatr 2009;155:469-74. [CrossRef]

22. Berardis S, Sokal E. Pediatric non-alcoholic fatty liver disease: An increasing public health issue. Eur J Pediatr 2014;173:131-9. [CrossRef]

23. Kitson MT, Roberts SK. D-livering the message: The importance of vitamin D status in chronic liver disease. J Hepatol 2012;57:897-909.

24. Fraser A, Longnecker P, Lawlor DA. Prevalence of elevated alanine aminotransferase among US adolescents and associated factors: NHANES 1999-2004. Gastroenterology 2007;133:1814-20. [CrossRef]

25. Bolland MJ, Grey AB, Ames RW, Mason BH, Horne AM, Gamble GD, et al. The effects of seasonal variation of 25-hydroxyvitamin D and fat mass on a diagnosis of Vitamin D sufficiency. Am J Clin Nutr 2007;86:959-64. [CrossRef] 